\title{
Reporter-Free Potentiometric Sensing of Boronic Acids and Their Reactions by Using Quaternary Ammonium Salt-Functionalized Polymeric Liquid Membranes
}

\author{
Xuewei Wang, ${ }^{\dagger, \ddagger}$ Dengfeng Yue, ${ }^{\S}$ Enguang $\mathrm{Lv}^{\dagger}{ }^{\dagger}$ Lei Wu, ${ }^{\dagger}$ and Wei Qin*, ${ }^{\dagger}$ \\ ${ }^{\dagger}$ Key Laboratory of Coastal Environmental Processes and Ecological Remediation, Yantai Institute of Coastal Zone Research (YIC), \\ Chinese Academy of Sciences (CAS); Shandong Provincial Key Laboratory of Coastal Environmental Processes, YICCAS, Yantai, \\ Shandong 264003, P. R. China \\ ${ }^{\ddagger}$ Graduate University of the Chinese Academy of Sciences, Beijing 100049, P. R. China \\ ${ }^{\S}$ College of Chemistry and Chemical Engineering, Yantai University, Yantai, Shandong 264003, P. R. China
}

\section{Supporting Information}

\begin{abstract}
The tremendous applications of boronic acids (BAs) in chemical sensing, medical chemistry, molecular assembly, and organic synthesis lead to an urgent demand for developing effective sensing methods for BAs. This paper reports a facile and sensitive potentiometric sensor scheme for heterogeneous detection of BAs based on their unexpected potential responses on quaternary ammonium salt-doped

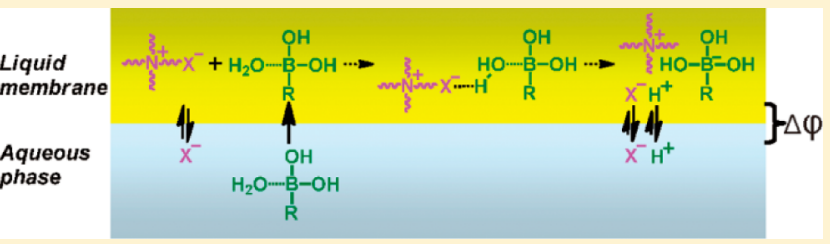
polymeric liquid membranes. ${ }^{11} \mathrm{~B}$ NMR data reveal that a quaternary ammonium chloride can trigger the hydrolysis of an electrically neutral BA in an aprotic solvent. Using the quaternary ammonium salt as the receptor, the BA molecules can be extracted from the sample solution into the polymeric membrane phase and undergo the concomitant hydrolysis. Such salttriggered hydrolysis generates $\mathrm{H}^{+}$ions, which can be coejected into the aqueous phase with the counterions (e.g., $\mathrm{Cl}^{-}$) owing to their high hydrophilicities. The perturbation on the ionic partition at the sample-membrane interface changes the phase boundary potential and thus enables the potentiometric sensing of BAs. In contrast to other transduction methods for BAs, for which labeled or separate reporters are exclusively required, the present heterogeneous sensing scheme allows the direct detection of BAs without using any reporter molecules. This technique shows superior detection limits for BAs $\left(\right.$ e.g., $1.0 \times 10^{-6}$ $\mathrm{M}$ for phenylboronic acid) as compared to previously reported methods based on colorimetry, fluorimetry, and mass spectrometry. The proposed sensing strategy has also been successfully applied to potentiometric indication of the BA reactions with hydrogen peroxide and saccharides, which allows indirect and sensitive detection of these important species.
\end{abstract}

B oronic acids (BAs) are a class of green compounds which have been extensively used as molecular recognition elements in chemical sensing, pharmaceutical agents in medical chemistry, building blocks in molecular assembly, and coupling reactants in organic synthesis. ${ }^{1}$ The expanding applications of BAs necessitate effective and facile sensing techniques to quantify them and characterize their reactions. Unfortunately, the BA moiety itself has no intrinsic UV-vis absorbance, fluorescence, or redox activities. BAs can even hardly yield molecular ion peaks in traditional mass spectrometry owing to their thermal instability. ${ }^{2}$ Reporter units such as fluorophores, chromophores, and redox active groups are usually labeled to BAs (the BA moiety is retained) to transduce recognition reactions with the BA moiety. ${ }^{3}$ However, the labeling processes are complicated, laborious, and expensive. More importantly, a large number of reporter-conjugated BAs suffer from problems of poor aqueous solubilities, which limits their applications especially in biological fluids. ${ }^{4}$ As an alternative, unlabeled reporter molecules (indicators) have been used whose optical or electrochemical properties can change upon their specific reactions with the $\mathrm{BA}$ moiety. ${ }^{5}$ However, these indicators usually bind to and thus destroy the reactive BA moiety, which may change the reaction rates and/or equilibrium of the primary reactions of BAs and induce difficulties in the in situ characterization of these reactions. ${ }^{5 a}$ So far, the direct sensing of BAs and their reactions without using any reagents either as labeled reporters or added indicators remains a significant challenge.

Polymeric liquid membrane electrodes (PLMEs) have been used as potentiometric ion-selective transducers for more than half a century. ${ }^{6}$ With attractive features of portability, low cost and energy consumption, ease of miniaturization and integration, and resistance to interferences from color and turbidity, PLMEs have been developed for detection of over 60 inorganics, organics, biological ions, and several classes of neutral species and gained great successes in many important fields such as blood electrolyte analyses, noninvasive microtests, and intracellular and extracellular ion measurements. ${ }^{7}$ However,

Received: December 30, 2013

Accepted: January 31, 2014

Published: January 31, 2014 
despite the well-known importance of BAs, PLMEs response to this class of compounds has never been reported. This may be due to the fact that a large number of BAs are mainly nonionic under the commonly used physiological $\mathrm{pH}$ conditions. Herein, we show that unexpected hydrolysis of neutral BAs can be triggered by using a quaternary ammonium salt in an aprotic solvent. The potential responses of neutral BAs are obtained at the PLMEs functionalized by the quaternary ammonium salt. Such responses enable direct potentiometric determination of BAs without special modification of them with reporter units, which dramatically simplifies the detection procedures. In addition, the proposed sensing strategy utilizes the heterogeneous hydrolysis of neutral BAs extracted into the waterimmiscible liquid membrane rather than the homogeneous BAindicator reactions in the sample phase, thus avoiding the indicator-induced change of the BA moiety.

Figure 1 shows the potential responses of a number of alkyl BAs and aryl BAs on a tetradodecylammonium chloride
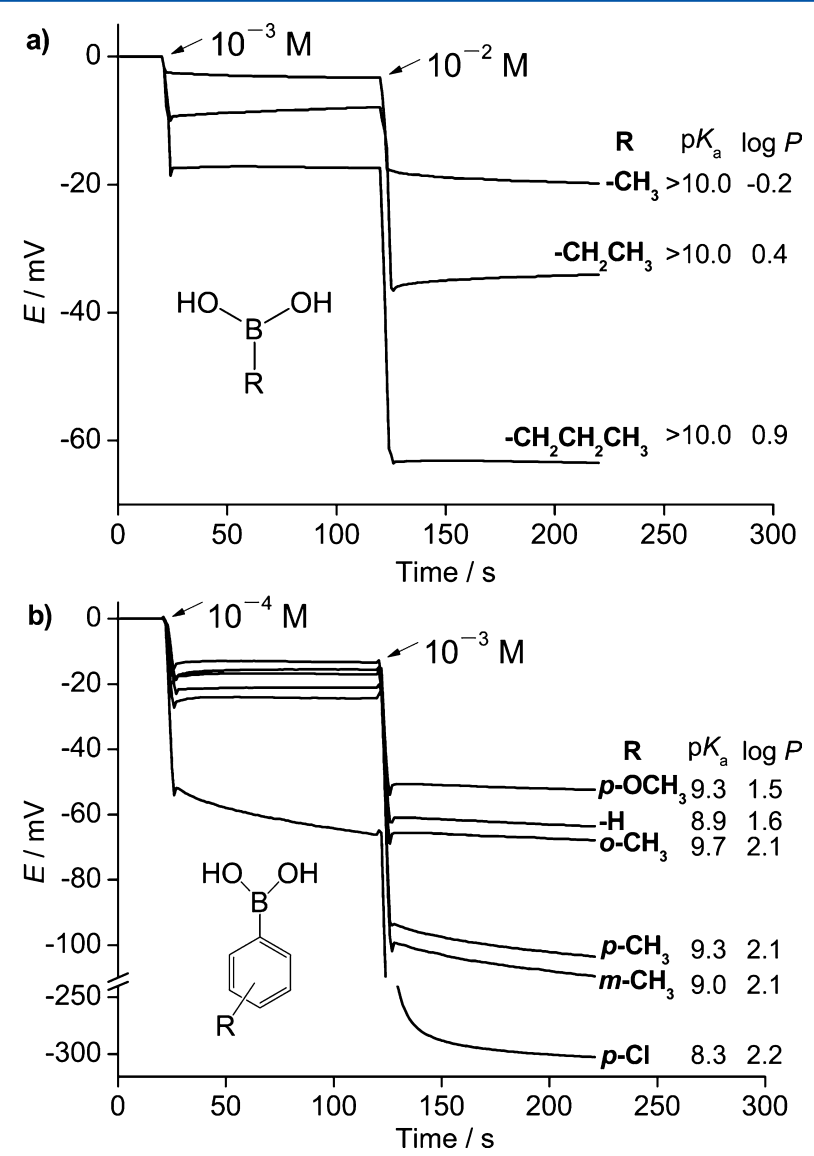

Figure 1. Potential responses to (a) three alkyl BAs in a $50 \mathrm{mM}$ HEPES buffer of $\mathrm{pH} 7.4$ and to (b) six aryl BAs in a $50 \mathrm{mM}$ HEPES buffer of $\mathrm{pH} 6.0$ at the $o$-NPOE-PVC-TDDA ${ }^{+} \mathrm{Cl}^{-}$membrane electrode. See references 8 for $\mathrm{p} K_{\mathrm{a}}$ of alkyl $\mathrm{BAs}$ and $5 \mathrm{a}$ and 9 for $\mathrm{p} K_{\mathrm{a}}$ of aryl BAs. All $\log P$ values in this paper were calculated by ACD/ Labs 12.

$\left(\mathrm{TDDA}^{+} \mathrm{Cl}^{-}\right)$-doped polymeric membrane. Since buffers with Lewis basic components (e.g., phosphate and citrate systems) may associate with BAs and partly ionize them, HEPES buffers without obvious interactions with BAs were employed. ${ }^{5 a}$ The $\mathrm{pH}$ values of the used HEPES buffers are 7.4 and 6.0 for the measurements of alkyl BAs and aryl BAs, respectively, to ensure they are dominantly nonionic (>99\%). As can be seen, both alkyl BAs and aryl BAs induce anionic potential responses in their neutral forms (see Figures S1 and S2, Supporting Information, for the potential-log $\mathrm{C}$ curves). Other similar compounds without the BA moiety (benzene and methyl-, methoxy-, and chloro-substituted benzene derivatives, $10^{-3} \mathrm{M}$ ) show negligible anionic potential responses (smaller than 1 $\mathrm{mV}$ ). Therefore, it can be concluded that the BA moiety is crucial to the potential responses as shown in Figure 1.

Electrically neutral species cannot directly induce potential responses at the interface between the aqueous phase and the polymeric liquid membrane. ${ }^{10}$ The most possible mechanism of neutral species-induced changes in the phase boundary potential is that they perturb the distribution of ionic species in the biphasic system. BAs can associate with anions via Lewis acid-base interactions. ${ }^{11}$ However, unlike the interactions with strong Lewis bases such as $\mathrm{F}^{-}, \mathrm{CN}^{-}$, and $\mathrm{AcO}^{-}$, the associations of BAs with $\mathrm{Cl}^{-}$are insignificant owing to the limited Lewis basicity of $\mathrm{Cl}^{-}{ }^{11,12}$ Another ion-involved reaction of the BA may be its solvolysis, which can be triggered by nucleophiles (e.g., amines) and generate the tetrahedral boronate ion. ${ }^{13}$ To examine if the $\mathrm{BA}$ undergoes solvolysis in the water-liquid membrane system, liquid-liquid extraction was conducted in the water- $\mathrm{CHCl}_{3}$ (or $\mathrm{CDCl}_{3}$ for $\mathrm{NMR}$ measurements) biphasic system. As shown in Figure 2, when neutral

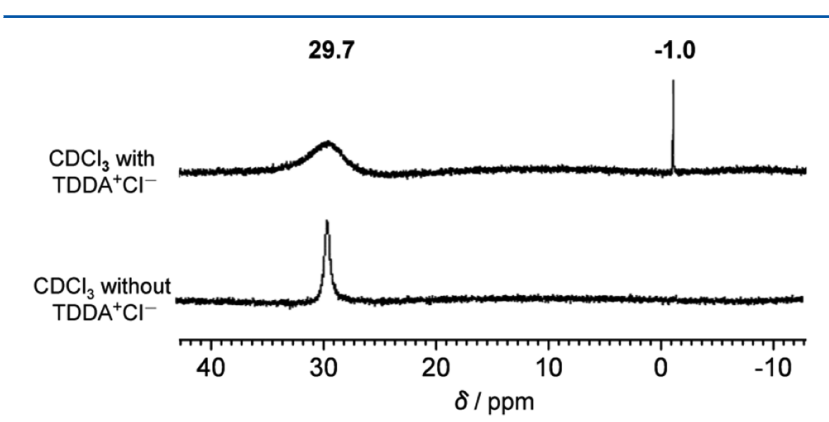

Figure 2. ${ }^{11} \mathrm{~B}$ NMR spectra of $\mathrm{CDCl}_{3}$ with and without $\mathrm{TDDA}^{+} \mathrm{Cl}^{-}$ $(20 \mathrm{mM})$ after extraction of PBA from an aqueous solution $(20 \mathrm{mM}$, $\mathrm{pH}$ 5.0).

phenylboronic acid (PBA) in water is extracted into pure $\mathrm{CDCl}_{3}$, the ${ }^{11} \mathrm{~B}$ NMR spectrum of the $\mathrm{CDCl}_{3}$ phase shows a peak at $29.7 \mathrm{ppm}$, which originates from the neutral PBA with the trigonal $\mathrm{sp}^{2}$ boron; ${ }^{14}$ however, when $\mathrm{CDCl}_{3}$ with $\mathrm{TDDA}^{+} \mathrm{Cl}^{-}$is used to extract neutral PBA, a peak at -1.0 ppm can be observed in addition to that at $29.7 \mathrm{ppm}$ in the ${ }^{11} \mathrm{~B}$ NMR spectrum. The large upfield peak shift indicates the generation of the anionic tetrahedral $\mathrm{sp}^{3}$ boron ${ }^{14,15}$ and most likely results from the hydrolysis of neutral PBA into its anionic boronate form. Experiments also show that the $\mathrm{pH}$ of the aqueous phase can be largely decreased (from 5.0 to 3.4) after extraction of the aqueous $\mathrm{PBA}$ into the $\mathrm{CHCl}_{3}$ phase containing $\mathrm{TDDA}^{+} \mathrm{Cl}^{-}$(see Table S1, Supporting Information), which indicates the generation of $\mathrm{H}^{+}$and thereby confirms the hydrolysis of $\mathrm{PBA}$ in the organic phase. Moreover, the $\mathrm{TDDA}^{+} \mathrm{Cl}^{-}$-doped $\mathrm{CHCl}_{3}$ shows a significantly increased extraction efficiency for PBA (64\%) as compared to pure $\mathrm{CHCl}_{3}$ (26\%) (see Table S1, Supporting Information), which further suggests that $\mathrm{PBA}$ undergoes a chemical reaction in the $\mathrm{TDDA}^{+} \mathrm{Cl}^{-}$-doped $\mathrm{CHCl}_{3}$ phase.

In contrast to the traditional understanding that $\mathrm{Cl}^{-}$cannot react significantly with BAs, to our knowledge, this paper shows for the first time the chloride salt-induced hydrolysis of BAs. It 
Scheme 1. Proposed Response Mechanism of a Neutral BA on the Liquid Membrane Electrode Doped with a Quaternary Ammonium Salt

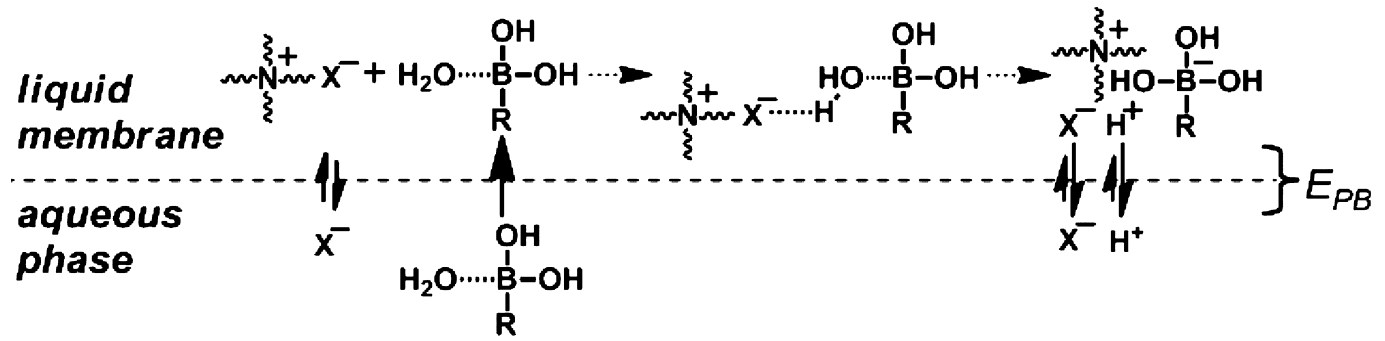

has been reported that quaternary ammonium salts can induce the similar proton dissociation processes for the phenolic hydroxyl group. ${ }^{7 \mathrm{c}}$ The hydrolysis of a BA is probably triggered by its $\mathrm{H}$-bonding to $\mathrm{Cl}^{-}$because $\mathrm{Cl}^{-}$is a nucleophile with a $\mathrm{H}$ bond basicity ${ }^{16}$ and could form a hydrogen bond with the $\mathrm{H}_{2} \mathrm{O}$ molecule "coordinated" on the BA. ${ }^{17}$ In addition, the cationic quaternary ammonium is expected to facilitate the hydrolysis reaction via formation of cooperative ion pairs with the hydrolyzed BA.

Since the $\mathrm{H}^{+}$ion generated by hydrolysis is hydrophilic, it is ejected into the aqueous phase from the organic phase together with the hydrophilic $\mathrm{Cl}^{-}$ion. Indeed, the concentration of $\mathrm{Cl}^{-}$ in the aqueous phase is increased upon the BA extraction (see Table S1, Supporting Information). The decrease in the concentration of $\mathrm{Cl}^{-}$in the polymeric liquid membrane and its increase in the aqueous phase could result in an anionic potential response according to the Nernstian equation. ${ }^{18}$ Notably, unlike traditional ion-selective electrodes with constant activities of primary ions in membranes, the BAsensitive electrode is not conditioned by target species before measurements and does not show a typical Nernstian response slope (Figures S1 and S2, Supporting Information).

The chloride salt was employed as the model receptor because $\mathrm{Cl}^{-}$is abundant in most biological fluids. $\mathrm{Cl}^{-}$would be prone to occupy the anionic sites in the membrane sensing layer no matter whether the counterion of the quaternary ammonium preincorporated in the membrane is $\mathrm{Cl}^{-}$or not. However, for membranes containing quaternary ammonium salts of other anions, potential responses can also be obtained but would be dependent on the $\mathrm{H}$-bond basicities and hydrophilicities of the anions (Figure S3, Supporting Information). On the other hand, the cation of the receptor must be lipophilic enough to hold the receptor salt in the organic membrane and thus ensure the receptor-induced BA hydrolysis (Figure S3, Supporting Information). On the basis of the above evidence, the response model of the neutral BA on the receptor-doped PLME can be illustrated in Scheme 1.

The sensitivities of the $\mathrm{TDDA}^{+} \mathrm{Cl}^{-}$-doped PLME toward different BAs were examined. Since the solvent polymeric membrane is a hydrophobic oil phase, the BAs with higher hydrophobicities would be extracted into the membrane more favorably and thus induce larger potential responses (Figure S4, Supporting Information). The acidity of the BA is another factor responsible for the potential response because it governs the BA hydrolysis in combination with the H-bond-basicity of the receptor anion (Figure S5, Supporting Information). For BAs tested in Figure 1, the potential responses follow the same rules based on lipophilicities $\left(p-\mathrm{CH}_{3} \mathrm{O}-\mathrm{PBA}<p-\mathrm{CH}_{3}-\mathrm{PBA}\right)$ and acidities $\left(o-\mathrm{CH}_{3}\right.$-PBA $<p-\mathrm{CH}_{3}$-PBA $<m-\mathrm{CH}_{3}$-PBA $<p$-Cl$\mathrm{PBA}$ ) of BAs. In addition, $\mathrm{Cl}^{-}$in the sample would decrease the potential response of BA by suppressing the ejection of $\mathrm{Cl}^{-}$ from the membrane into the sample.

For direct detection of BAs, the detection limits of the potentiometric sensor are in the ranges of $10^{-6}-10^{-5}$ and $10^{-7}-10^{-6} \mathrm{M}$ for alkyl and aryl BAs, respectively (Figures S1 and S2, Supporting Information). Taking PBA for example, the obtained detection limit of $1.0 \times 10^{-6} \mathrm{M}(3 \sigma)$ is at least 2 orders of magnitude lower than those of previously reported methods based on colorimetry, fluorimetry, and mass spectrometry. ${ }^{2 \mathrm{~b}, 5 \mathrm{c}, \mathrm{d}}$ The linear detection range for PBA is 5.0 $\times 10^{-5}$ to $5.0 \times 10^{-3} \mathrm{M}$ (Figure S6, Supporting Information), and the response time varies from $10 \mathrm{~s}$ to $10 \mathrm{~min}$ depending on the tested PBA concentration (Figure S7, Supporting Information). The potential responses are fully reversible via regeneration of the electrode after each measurement (Figure S8, Supporting Information).

As a preliminary application, the BA-sensitive PLME was used to indicate the oxidation of an aryl $\mathrm{BA}$ by $\mathrm{H}_{2} \mathrm{O}_{2}$ into a phenolic product, ${ }^{19}$ which has been widely utilized for the detection of $\mathrm{H}_{2} \mathrm{O}_{2}$. 4-Benzylphenylboronic acid (4-BPBA, $\log P$ $\left.=3.6, \mathrm{p} K_{\mathrm{a}}=8.7\right)$ was chosen for the present work because it shows a large anionic potential response on the membrane. As illustrated in Figure S9, Supporting Information, a potential increase can be induced by oxidation (i.e., consumption) of 4BPBA with $\mathrm{H}_{2} \mathrm{O}_{2}$ in the HEPES buffer of $\mathrm{pH} 7.4 .^{20}$ The potential change measured at 10 min after addition of $\mathrm{H}_{2} \mathrm{O}_{2}$ was used for quantification. Experiments showed that $\mathrm{H}_{2} \mathrm{O}_{2}$ could be detected with a linear range of $5 \times 10^{-5}$ to $5 \times 10^{-4} \mathrm{M}$ and a detection limit of $2 \times 10^{-5} \mathrm{M}$.

It is known that the most commonly used reactions of BAs might be their esterification reactions with diol-containing compounds such as saccharides, catecholamines, and glycoproteins. ${ }^{1}$ Among them, the reactions with saccharides have attracted special attention because they allow the development of enzyme-free saccharide sensors. Using 4-BPBA as the substrate as well, its reaction with $\mathrm{D}$-fructose (a saccharide showing a high binding affinity with the BA moiety $)^{5 \mathrm{a}}$ in a $\mathrm{pH}$ 7.4 HEPES buffer can induce a quick potential reversal (Figure S10, Supporting Information). This is probably due to the fact that the product from the complexation between the lipophilic 4-BPBA and the hydrophilic saccharide ( $\log P$ (D-fructose) $=$ -1.5 ) is expected to be much less lipophilic than 4-BPBA and cannot be readily extracted into the polymeric membrane.

An aryl BA with a lower $\mathrm{p} K_{\mathrm{a}}$ can bind more strongly with saccharides at the physiological $\mathrm{pH}$ and show higher sensitivities for saccharide detection. ${ }^{21}$ Therefore, 2-fluorobiphenyl boronic acid (2-FBPBA, $\mathrm{pK} K_{\mathrm{a}}=7.5$ ) was further examined as the probe for saccharides to gain a higher detection sensitivity. In analogy to the esterification reaction of 4-BPBA, the reaction of 2-FBPBA $(\log P=3.9)$ with $\mathrm{D}$-fructose 
also decreases the lipophilicity of the parent BA and induces a reversed potential response (Figure 3). Indeed, the sensitivity

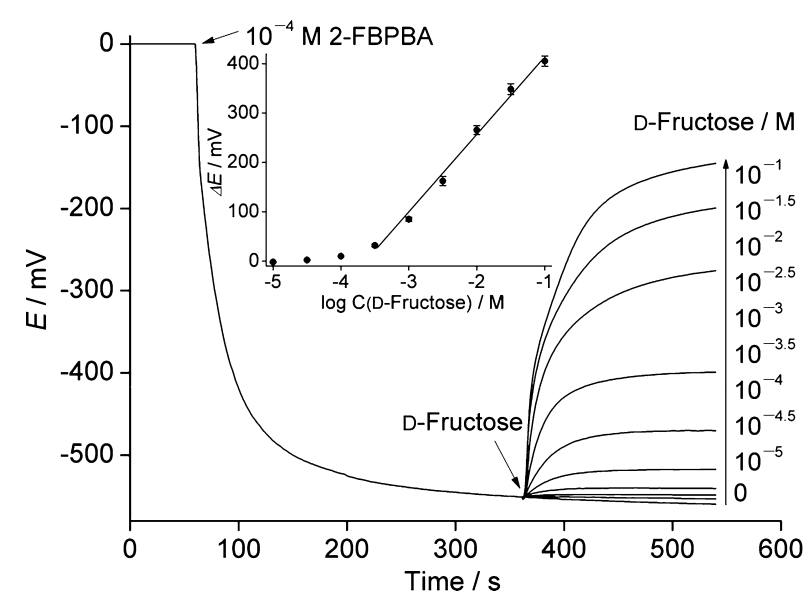

Figure 3. Potential responses to 2-FBPBA and its reactions with different concentrations of D-fructose in the HEPES buffer $(50 \mathrm{mM}$, $\mathrm{pH}$ 7.4) at the $\mathrm{TDDA}^{+} \mathrm{Cl}^{-}$-doped PLME. The inset shows the calibration curve for detection of $\mathrm{D}$-fructose. Each error bar represents one standard deviation for three measurements.

of the membrane electrode using 2-FBPBA $\left(275.0 \mathrm{mV}\right.$ for $10^{-2}$ $\mathrm{M}$ D-fructose) could be dramatically improved as compared to that obtained by using 4-BPBA (97.4 mV in Figure S10, Supporting Information). Using the potential change measured at $3 \mathrm{~min}$ after addition of $\mathrm{D}$-fructose for quantification, a linear range of $5 \times 10^{-4}$ to $1 \times 10^{-1} \mathrm{M}$ and a detection limit of $1 \times$ $10^{-5} \mathrm{M}(3 \sigma)$ for $\mathrm{D}$-fructose were obtained. This detection limit is lower than those obtained by most fluorescent and electrochemical methods based on the similar BA recognition. ${ }^{3,22}$ The sensitivity of the proposed system is also much higher than that of the previously reported boronic acid-based potentiometric sensor for saccharides (i.e., $250 \mathrm{vs} 4.4 \mathrm{mV}$ for 10 $\mathrm{mM}$ D-fructose). ${ }^{2 \mathrm{a}}$ For other saccharides, the detection sensitivities are proportional to the binding constants with the BA moiety (Figure S11, Supporting Information), which further confirms that the potential responses are induced by the esterification reactions.

In conclusion, we have developed BA-sensitive membrane electrodes for direct potentiometric sensing of BAs and their reactions in a reporter-free manner. The quaternary ammonium salts have been found to induce hydrolysis of BAs in an aprotic solvent and can serve as the molecular receptors for BAs. The present PLMEs are cost-effective, easy to prepare, less invasive, and regenerable. The proposed sensing principle can be extended to develop liquid membrane-based optrodes and other liquid-liquid interface-based electrodes for BAs. Moreover, the salt-based receptors hold promise as carriers in transmembrane delivery of BA or BA-labeled drugs.

\section{ASSOCIATED CONTENT}

\section{S Supporting Information}

Experimental details and other figures. This material is available free of charge via the Internet at http://pubs.acs.org.

\section{AUTHOR INFORMATION}

\section{Corresponding Author}

*E-mail: wqin@yic.ac.cn.

\section{Notes}

The authors declare no competing financial interest.

\section{ACKNOWLEDGMENTS}

This work was financially supported by the Instrument Developing Project of the Chinese Academy of Sciences (YZ201161), the National Natural Science Foundation of China (41176081), and the Taishan Scholar Program of Shandong Province (TS20081159). We thank Dr. Xiuli Yin for her help with NMR measurements.

\section{REFERENCES}

(1) (a) Hall, D. G. Boronic Acids: Preparation and Applications in Organic Synthesis, Medicine and Materials, 2nd ed.; Wiley-VCH: Weinheim, 2012. (b) Bull, S. D.; Davidson, M. G.; Elsen, J. M.; Fossey, J. S.; Jenkins, A. T.; Jiang, Y. B.; Kubo, Y.; Marken, F.; Sakurai, K.; Zhao, J.; James, T. D. Acc. Chem. Res. 2013, 46, 312-326.

(2) (a) Llewellyn, G.; Stein, B. K. Boronic Acid Analysis by Mass Spectrometry-I: Cis-diol Derivatisation for EI and CI Analysis: Application Note No 6; EPSRC National Mass Spectrometry Centre: Swansea, 2008, http://www.nmssc.ac.uk/documents/NMSSC-AN6BOH2-EG.pdf. (b) Flender, C.; Leonhard, P.; Wolf, C.; Fritzsche, M.; Karas, M. Anal. Chem. 2010, 82, 4194-4200.

(3) Egawa, Y.; Seki, T.; Takahashi, S.; Anzai, J. Mater. Sci. Eng., C 2011, 31, 1257-1264.

(4) (a) Cesare, N. D.; Lakowicz, J. R. J. Phys. Chem. A 2001, 105, 6834-6840. (b) Xu, W. Z.; Huang, Z. T.; Zheng, Q. Y. Tetrahedron Lett. 2008, 49, 4918-4921. (c) Shao, M.; Zhao, Y. Tetrahedron Lett. 2010, 51, 2508-2511. (d) Liu, Y.; Deng, C.; Tang, L.; Qin, A.; Hu, R.; Sun, J. Z.; Tang, B. Z. J. Am. Chem. Soc. 2011, 133, 660-663. (e) Huang, Y. J.; Ouyang, W. J.; Wu, X.; Li, Z.; Fossey, J. S.; James, T. D.; Jiang, Y. B. J. Am. Chem. Soc. 2013, 135, 1700-1703.

(5) (a) Springsteen, G.; Wang, B. Tetrahedron 2002, 58, 5291-5300. (b) Barder, T. E.; Buchwald, S. L. Org. Lett. 2007, 9, 137-139. (c) Lawrence, K.; Flower, S. E.; Kociok-Kohn, G.; Frost, C. G.; James, T. D. Anal. Methods 2012, 4, 2215-2217. (d) Lim, J.; Nam, D.; Miljanic, O. Š. Chem. Sci. 2012, 3, 559-563. (e) Liu, H.; Crooks, R. M. Anal. Chem. 2013, 85, 1834-1839.

(6) Buhlmann, P.; Chen, L. D. In Supramolecular Chemistry: From Molecules to Nanomaterials; Steed, A. W., Gale, P., Eds.; John Wiley \& Sons, Ltd: New York, 2012.

(7) (a) Smith, P. J. S. Nature 1995, 378, 645-646. (b) Bühlmann, P.; Pretsch, E.; Bakker, E. Chem. Rev. 1998, 98, 1593-1687. (c) Ito, T.; Radecka, H.; Tohda, K.; Odashima, K.; Umezawa, Y. J. Am. Chem. Soc. 1998, 120, 3049-3059. (d) Guenat, O. T.; Generelli, S.; de Rooij, N. F.; Koudelka-Hep, M.; Berthiaume, F.; Yarmush, M. L. Anal. Chem. 2006, 78, 7453-7460. (e) Bakker, E.; Pretsch, E. Angew. Chem., Int., Ed. 2007, 46, 5660-5668. (f) Ding, J. W.; Qin, W. J. Am. Chem. Soc. 2009, 131, 14640-14641. (j) Liang, R. N.; Song, D. A.; Zhang, R. M.; Qin, W. Angew. Chem., Int. Ed. 2010, 49, 2556-2559.

(8) (a) Babcock, L.; Pizer, R. Inorg. Chem. 1980, 19, 56-61. (b) Weisbecker, C. S.; Merritt, M. V.; Whitesides, G. M. Langmuir 1996, 12, 3763-3772.

(9) $\mathrm{p} K_{\mathrm{a}}$ values were determined by their UV absorption changes from non-ionic boronic acid form to anionic boronate form in an aqueous solution of $0.1 \mathrm{M} \mathrm{NaCl}$. For details, see: Soundararajan, S.; Badawi, M.; Kohlrust, C. M.; Hageman, J. H. Anal. Biochem. 1989, 178, 125134.

(10) (a) Espadas-Torre, C.; Bakker, E.; Barker, S.; Meyerhoff, M. E. Anal. Chem. 1996, 68, 1623-1631. (b) Bühlmann, P.; Hayakawa, M.; Ohshiro, T.; Amemiya, S.; Umezawa, Y. Anal. Chem. 2001, 73, 31993205. (c) Wang, X.; Qin, W. Chem. Commun. 2012, 48, 4073-4075. (d) Wang, X.; Qin, W. Chem.-Eur. J. 2013, 19, 9979-9986.

(11) Nishiyabu, R.; Kubo, Y.; James, T. D.; Fossey, J. S. Chem. Commun. 2011, 47, 1106-1123.

(12) Dusemund, C.; Samankumara Sandanayake, K. R. A.; Shinkai, S. J. Chem. Soc., Chem. Commun. 1995, 333-334. 
(13) (a) Zhu, L.; Shabbir, S. H.; Gray, M.; Lynch, V. M.; Sorey, S.; Anslyn, E. V. J. Am. Chem. Soc. 2006, 128, 1222-1232. (b) Kataoka, K.; James, T. D.; Kubo, Y. J. Am. Chem. Soc. 2007, 129, 15126-15127.

(c) Kataoka, K.; Okuyama, S.; Minami, T.; James, T. D.; Kubo, Y. Chem. Commun. 2009, 13, 1682-1684.

(14) Tomsho, J. W.; Benkovic, S. J. J. Org. Chem. 2012, 77, 20982106.

(15) Matsumura, T.; Iwatsuki, S.; Ishiharaa, K. Inorg. Chem. Commun. 2005, 8, 713-716.

(16) Zhao, H. In Ionic Liquids in Biotransformations and Organocatalysis: Solvents and Beyond;Domínguez de María, P., Ed; John Wiley \& Sons, Hoboken, NJ, 2012.

(17) A downfield shift (from 7.74 to $7.84 \mathrm{ppm}$ ) of $\mathrm{H}_{\text {ortho }}$ of PBA was observed after addition of $\mathrm{TDDA}^{+} \mathrm{Cl}^{-}$into the $\mathrm{CF}$ containing $\mathrm{PBA}$ in the ${ }^{1} \mathrm{H}$ NMR spectrum, which suggests the formation of $\mathrm{H}$-bond.

(18) Bakker, E.; Bühlmann, P.; Pretsch, E. Talanta 2004, 63, 3-20.

(19) Kuivila, H. G. J. Am. Chem. Soc. 1954, 76, 870-874.

(20) The product is 4-benzylphenol whose potential response $(-248$ $\mathrm{mV}$ for $\left.10^{-4} \mathrm{M}\right)$ is smaller than that of 4-BPBA $\left(-392 \mathrm{mV}\right.$ for $10^{-4}$ $\mathrm{M})$.

(21) Singhal, R. P.; Ramamurthy, B.; Govindraj, N.; Sarwar, Y. J. Chromatogr. 1991, 543, 17-38.

(22) (a) Shoji, E.; Freund, M. S. J. Am. Chem. Soc. 2002, 124, 1248612493. (b) Zhai, J.; Pan, T.; Zhu, J.; Xu, Y.; Chen, J.; Xie, Y.; Qin, Y. Anal. Chem. 2012, 84, 10214-10220. 